\section{Tafazzin gene mutations are uncommon causes of dilated cardiomyopathy in adults}

\author{
Matthew Taylor, ${ }^{1}$ Dobromir Slavov, ${ }^{1}$ \\ Ernesto Salcedo, ${ }^{1}$ Xiao Zhu, ${ }^{1}$ \\ Deborah Ferguson, ${ }^{1}$ Jean Jirikowic, ${ }^{1}$ \\ Andrea Di Lenarda, ${ }^{2}$ Gianfranco Sinagra, ${ }^{2}$ \\ Luisa Mestroni' \\ 1 University of Colorado Denver, Aurora \\ CO, USA; ${ }^{2}$ Ospedale Cattinara and \\ University of Trieste, Trieste, Italy
}

\section{Abstract}

Barth syndrome is an X-linked genetic condition featuring neutropenia, skeletal myopathy, and dilated cardiomyopathy in boys due to tafazzin (TAZ) mutations. Pure dilated cardiomyopathy without other features of Barth syndrome may also result from $T A Z$ mutations and survival into adulthood has been described. Although $T A Z$ testing is routinely included in dilated cardiomyopathy panels in adults, the prevalence of $T A Z$ mutations in the adult population, including women who may be at risk to develop later onset disease due to $T A Z$ mutations, has not been measured. We screened 292 families with dilated cardiomyopathy ( 209 male and 83 female probands) for TAZ mutations using denaturing high-performance liquid chromatography and sequence analysis. Putative mutations were evaluated based on standard criteria including screening available relatives and healthy controls and for effects on splicing efficiency in the case of one intronic variant. Two variants suspicious for being pathogenic were found in two unrelated families (c.387T >C, Phe128Ser and c.507C >T, Leu169Leu). The Phe128Ser variant had been previously reported as a pathogenic mutation; however we determined that this variant is instead a rare polymorphism restricted to African Americans. The Leu169Leu variant was detected in a male patient and altered RNA processing in our minigene assay supporting a pathogenic role. No mutations in female subjects were detected. Tafazzin mutations were rare in our population of adults with dilated cardiomyopathy and none were found in females. Our findings indicate that genetic testing for tafazzin should not be routinely performed in dilated cardiomyopathy as suggested by current guidelines. Furthermore, the Phe128Ser variant is not pathogenic, but likely represents a benign polymorphism in persons of African American ancestry.

\section{Introduction}

Barth syndrome is a rare, genetic disorder characterized by the phenotype of neutropenia, skeletal muscle myopathy, growth retardation, and dilated cardiomyopathy in boys. Mutations in TAZ (Xq28), which encodes tafazzin, an acyltransferase protein of the outer mitochondrial membrane, cause the disease. Cardiac features usually presents in infancy with dilated cardiomyopathy, which is sometimes detected in the third trimester or within the first few days of life. ${ }^{1}$ Components of left-ventricular noncompaction cardiomyopathy are present in half of cases. ${ }^{2}$ Treatment with cardiac transplantation has been successful in a number of cases. ${ }^{3,4}$ The majority of patients in the Barth Syndrome foundation database (www.barthsyndrome.org) are children, reflecting the pediatric nature of presentation and diagnosis. One relatively large series $(n=34)$ noted a mean age of cardiomyopathy diagnosis of 5.5 months. ${ }^{2}$

Interestingly, cardiac dysfunction may improve in some survivors and treatment of infections and early cardiac disease may contribute to improved survival to adulthood. Adults with Barth syndrome are known with the age range of living individuals extending up to 49 years with improvement of extra-cardiac manifestations. ${ }^{1,5,6}$ However, adult cases of Barth syndrome are not well described and no systematic study of an adult cardiomyopathy population has been previously performed. Similarly, data on whether female carriers of $T A Z$ mutations could present with later-onset cardiomyopathy without neutropenia and skeletal myopathy are not available. Skewed Xinactivation has been demonstrated in carrier females suggesting that selection against cells with a mutant $T A Z$ occurs and abnormal echocardiogram findings in a female $T A Z$ mutation carrier have been described. ${ }^{6,7}$ Recent guidelines for the evaluation of genetic forms of cardiomyopathy recommend $T A Z$ screening in dilated cardiomyopathy and $T A Z$ DNA sequencing is included in the currently available dilated cardiomyopathy genetic testing panels in the United States. ${ }^{8}$ We hypothesized that $T A Z$ mutations could contribute to dilated cardiomyopathy diagnosed in adults and that based on X-inactivation data and examples from other X-linked cardiomyopathies that women might present with a cardiac phenotype as adults. To address these questions and provide data for accurate genetic counseling about the likelihood of TAZ mutations causing dilated cardiomyopathy in adults we tested 292 dilated cardiomyopathy families for TAZ mutations.
Correspondence: Matthew Taylor, 12700 East 19th Avenue, Room 8022, Mailstop F442, Aurora, C0, 80045, USA.

Tel. +1.303.724.1400 - Fax: 1.303.724.0858

E-mail: matthew.taylor@ucdenver.edu

Key words: dilated cardiomyopathy, tafazzin, genetics, Barth syndrome, neutropenia, genetic counseling.

Funding: Barth Syndrome Foundation (www.barthsyndrome.org).

Contributions: the authors contributed equally.

Conflict of interest: the authors report no conflicts of interest.

Received for publication: 18 March 2011. Accepted for publication: 9 June 2011.

This work is licensed under a Creative Commons Attribution NonCommercial 3.0 License (CC BYNC 3.0).

(C) Copyright M. Taylor et al., 2011

Licensee PAGEPress, Italy

Cardiogenetics 2011; 1:e4

doi:10.4081/cardiogenetics.2011.e4

\section{Materials and Methods}

Patients with dilated cardiomyopathy were studied from the Familial Cardiomyopathy Registry, a multicenter, two-decade-long ongoing project studying human hereditary cardiomyopathies. Probands from 292 unrelated families with dilated cardiomyopathy were tested (209 male and 83 female adults). Ninety-three percent of the subjects were Caucasian ascertained from Northern Italy and Colorado. The diagnosis of dilated cardiomyopathy was made according to published criteria. ${ }^{9}$ Extensive clinical data from patients and family included: medical history, family history, physical examination, electrocardiogram, and echocardiogram data. Families with obvious male-to-male transmission of a cardiomyopathy phenotype were excluded from this study. Informed consent was obtained from living subjects and the protocol was approved by the University of Colorado institutional review board. Mutation screening was done using denaturing high-performance liquid chromatography (DHPLC) with the Transgenomic WAVE mutation detection system (Transgenomic Inc., Omaha, NE, USA). Exon and surrounding peri-exonic regions for all 11 reported exons (NT_167198.1) were amplified by polymerase chain reaction (primer sequences available upon request) and were screened for mutations by DHPLC for the presence of heteroduplexes. Samples from male subjects were mixed (1:1 ratio) with wild-type control DNA to 
favor the formation of heteroduplexes. Abnormal DHPLC elution profiles underwent DNA sequencing (ABI 3730 DNA Sequencer, Applied Biosystems, Foster City, CA, USA) and the results were compared to consensus published $T A Z$ sequence and single-nucleotide polymorphism (SNP) databases. Unique variants were also compared against the Barth Syndrome Foundation mutation database (April 2010 version; www.barthsyndrome.org) and dbSNP. Potential mutations were further evaluated for evidence of segregation within the pedigree, presence in ethnically similar controls, conservation of amino acid sequence (nonsynonymous variants), and effects on splicing (synonymous variants). Effects on splicing efficiency (L169L mutation) were investigated using a minigene approach in HEK and HELA cells as previously described. ${ }^{10}$ Putative mutations were screened in ethnically similar controls by pyrosequencing (PSQ 96MA, Biotage AB, Uppsala, Sweden). For the F128S mutation found in an African American patient, 91 additional African American control samples from the Coriell Institute (Camden, NJ, USA) were also screened.

\section{Results}

Familial dilated cardiomyopathy, defined by pedigrees with two or more affected family members, was present in 159 families (54\%), while 133 (46\%) families were classified as sporadic based on just a single affected patient in the family history. The average age of diagnosis of cardiomyopathy was 42.3 (standard deviation \pm 14.5 ) years and was similar for males and females and the average left-ventricular ejection fraction for the cohort was $33 \%( \pm 13.8 \%)$. None of the families were ascertained on the basis of having a prior diagnosis of Barth syndrome, left-ventricular noncompaction, or known neutropenia. Cardiolipin levels and neutrophil counts were not available on any of the subjects in the study database. Two novel variants suspicious for being pathogenic mutations were detected in two unrelated male probands (c.383T $>C$, Phe128Ser and c.507C>T, Leu169Leu; NM_000116; Figure 1). Neither subject had any echocardiographic features of left-ventricular noncompaction.

The non-synonymous exon 5 c.383 T>C (hereafter, Phe128Ser) variant predicts the replacement of a non-polar hydrophobic phenylalanine with a smaller hydrophilic polar serine and was found in an African American man who was diagnosed with dilated cardiomyopathy at age 32 years. The affected patient had three living first-degree female relatives, but none were available for study. Our initial interpretation was that the Phe128Ser variant represented a recurrent pathogenic mutation since it had been previously reported as causing Barth syndrome. ${ }^{11}$ This Phe128Ser variant was absent in 89 available control samples ethnically similar to our overall study population, which was predominantly Caucasian and is also absent from the initial release of the 1000 genome project data of 629 individuals (www.1000genomes.org). Since our patient was African American, a group not well-represented in our standard control samples, we then screened 91 African American control samples. Of these ethnically matched samples, the Phe128Ser variant was detected in 7 heterozygous samples and 1 homozygous female sample (mean age 33.4 years; 37 years in case of homozygous sample); none of the 16 male control samples $(9.6 \%$ of the total alleles) had the Phe128Ser variant. Although we cannot exclude a low-penetrant mutation that manifests solely in males, it appears that this variant is non-pathogenic with a prevalence of 5.4\% in African American alleles. This finding highlights the value of obtaining ethnically similar controls when interpreting recurrent mutations. The c.507C > T (Leu169Leu) synonymous variant is located in exon 6 and was detected in an isolated male patient with dilated cardiomyopathy. Screening of 89 controls detected the variant in just two heterozygous female controls and the variant was absent from the 1000 genomes dataset. A nonsynonymous mutation at this residue (Leu169His) is reported in the Barth Syndrome Foundation mutation database (April 2010 version). Since the $c .507 \mathrm{C}>\mathrm{T}$ variant was found in a hemizygous male, we performed a minigene assay using a construct with $T A Z$ exons 6,7 , and 8 to see if alternative splicing occurred due to this variant. The variant $\mathrm{T}$ allele led to visibly decreased product containing normally spliced exon 6 as compared to the wild-type $\mathrm{C}$ allele (Figure 2). Normalized against the housekeeping Glyceraldehyde-3-phosphate dehydrogenase gene, the variant $\mathrm{T}$ allele product was reduced by $30 \%$ compared to the wild-type product, findings compatible with reduction of transcript levels which could lead to reduced tafazzin protein. Although the variant is rarely present in control samples from females, it remains suspicious for a pathogenic variant due to its absence in male control samples and the observed altered RNA processing in vitro compared to the wild-type allele.

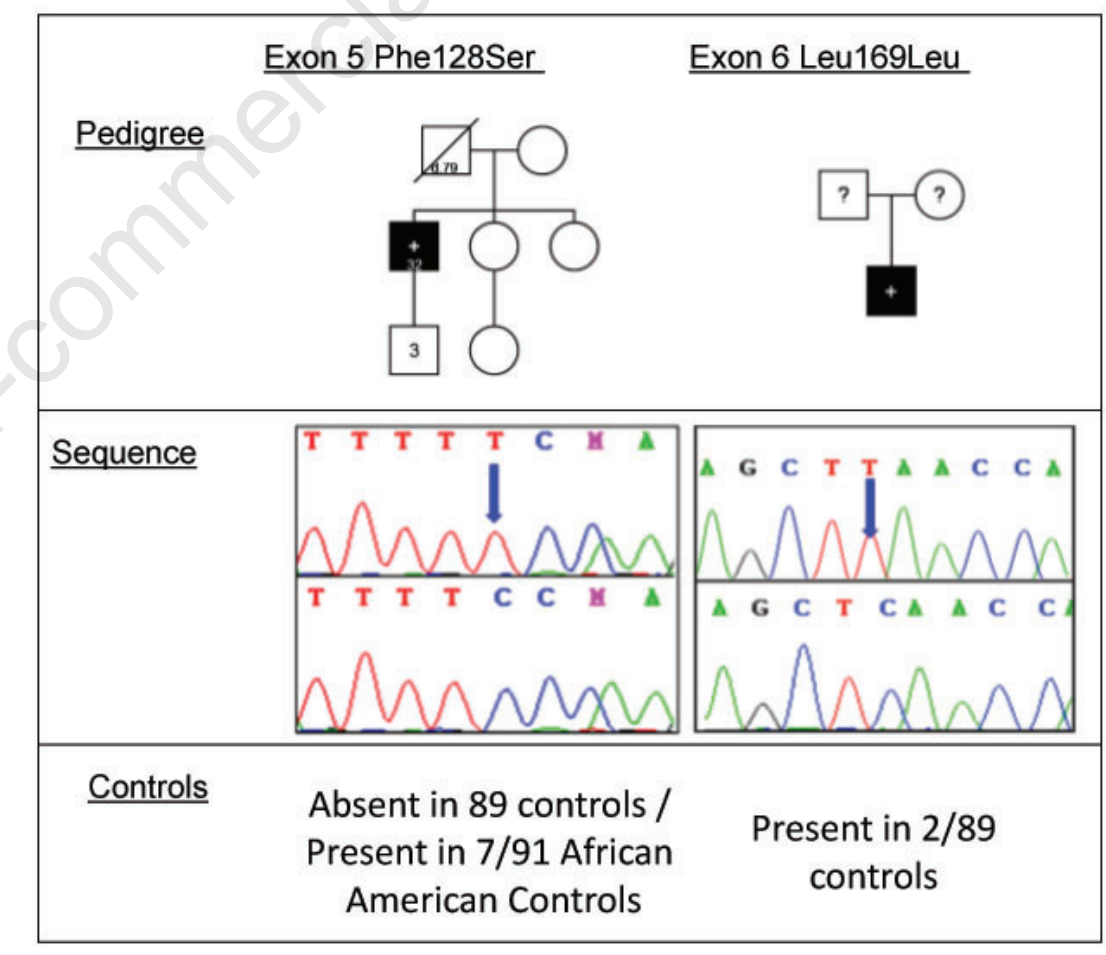

Figure 1. DNA variants detected in this study: The pedigrees, sequencing chromatograms, and results of screening controls are displayed. Males and females are represented by squares and circles in the pedigrees, respectively. Black and grey shading indicate confirmed and suspected dilated cardiomyopathy and/or heart failure respectively. Variant status is indicated by ' + '. Dates of cardiomyopathy diagnosis and death (d.) are indicated. '?' indicates no clinical data. The sequence of the mutations (top; blue arrows) and wild-type (bottom) DNA are displayed. 


\section{Discussion}

Barth syndrome has been best described in boys with the triad of dilated cardiomyopathy, skeletal muscle disease, and neutropenia. Reports of prolonged survival into adulthood are found in the literature and although women so far have been reported as asymptomatic, skewed X-inactivation silencing the wild-type X-chromosome could in theory lead to biological consequences of $T A Z$ mutations in female carriers. Others have reported that the Barth phenotype in boys may improve or resolve in some cases, which led us to speculate that recurrence of disease could be possible. ${ }^{5,6}$ Recently published guidelines for the evaluation of hereditary cardiomyopathy include $T A Z$ as a gene for clinical genetic testing in spite of the fact that a clear picture of $T A Z$ mutations in adult cardiomyopathy has not been presented. Our report clarifies the clinical contribution of $T A Z$ mutations in adult dilated cardiomyopathy. First, TAZ mutations are clearly a very rare cause of idiopathic dilated cardiomyopathy in adult males suggesting that Barth syndrome does not commonly manifest in adulthood as idiopathic dilated cardiomyopathy. The c.507C $>$ T variant present in 1 of 211 males (0.5\%) did affect splicing in our study supporting a pathogenic role. Nonsense mutations have been described as leading to more severe phenotypes and it is possible that c.507C $>\mathrm{T}$ variant substantially reduces levels of normally spliced product and leads to a reduction in native tafazzin protein. ${ }^{12}$ Unfortunately, we were unable to obtain any additional samples from this patient to test RNA or protein levels. The finding of this variant in two female controls is interesting and their lack of symptoms could be due to adequate levels of tafazzin from the normal allele. As our study did not identify any pathogenic mutations in women with cardiomyopathy we conclude that TAZ mutations are not a common cause of cardiac disease in adult women. While carrier women of other X-linked cardiomyopathies such as Duchenne muscular dystrophy and Danon disease may present with later onset cardiomyopathies, our data indicate that women carrying $T A Z$ mutations, discovered after the birth of a son with Barth syndrome, can be reassured and counseled about a low risk of themselves developing cardiomyopathy. ${ }^{13,14}$ Lastly, our data suggest that the Phe128Ser mutation previously reported as pathogenic, is most likely a common polymorphism in African Americans rather than a disease-causing mutation. Examples of genetic variants being restricted to certain ethnic groups and having functional significance have been reported, ${ }^{15-17}$ however our classification of Phe128Ser as likely benign is conservative and aligns with current clinical diagnostic

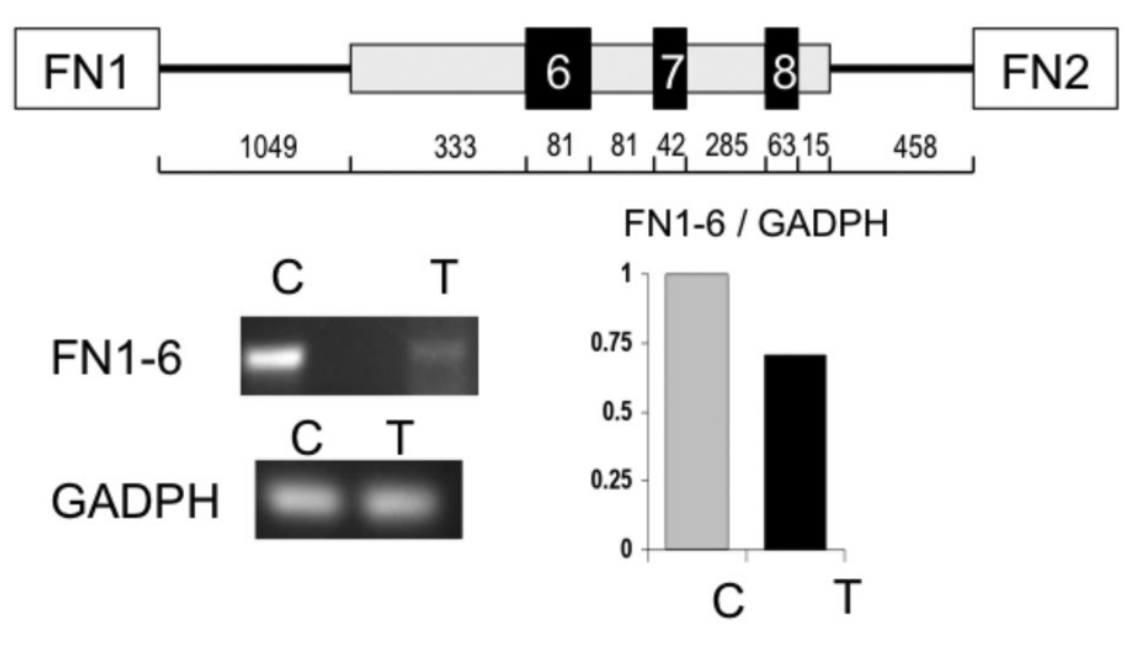

Figure 2. Minigene assay: Top- A construct containing TAZ exons 6, 7, and 8 were inserted into a minigene between two fibronectin exons. TAZ exons and introns (grey) indicated by numbered black boxes; basepair length indicated by numbers below the figure. Bottom- Mutant (T) and wildtype (C) minigene constructs were transfected into HEK (data shown) and HELA cells, RNA was extracted and made into cDNA, and the amount of product containing the correctly spliced in exon 6 product containing the $\mathrm{T}$ or $\mathrm{C}$ alleles was measured. In the case of the $T$ allele, reduced exon- 6 containing product was obtained as compared to the wild-type $C$ allele shown in the gel and in the graph as normalized against the GADPH housekeeping gene. FN, fibronectin exon; FN1-6, product containing correctly spliced in FN1 exon and TAZ exon 6; GADPH, glyceraldehyde-3phosphate dehydrogenase.

laboratory practice where novel variants found in control populations are reported as benign. Additional studies including functional data on the consequence of Phe128Ser would be required before a pathogenic role for Phe128Ser could be considered.

While our population was well-phenotyped and relatively large, it does represent a group recruited through a familial cardiomyopathy study and we cannot exclude any recruitment bias in our subjects. The large size of our population makes $T A Z$ mutations an unlikely cause of dilated cardiomyopathy. As Barth syndrome predominantly affects males, when available we selected samples from males subjects for $T A Z$ screening and this led to an under-representation of females (28\%) being tested in our cohort. It remains possible that $T A Z$ mutations could rarely contribute to dilated cardiomyopathy in other adult cardiomyopathy populations, including any cases where specific features of Barth syndrome such at neutropenia coexist with the cardiac phenotype. Furthermore, as survival of pediatric Barth syndrome due to $T A Z$ mutations improves it is conceivable that adults with the dilated phenotype will become part of the adult cardiomyopathy population. An additional limitation of our data is that the Leu169Leu mutation showed in vitro evidence of a functional consequence on RNA processing, however we were unable to obtain any RNA or protein sample from the patient to further validate this finding. Our approach used denaturing highperformance liquid chromatography, which has known sensitivity limitations, and it is possible that we failed to detect all $T A Z$ variants. The advent of next-generation DNA sequencing technologies, which can achieve higher sensitivity if sufficient sequencing depth is used, would improve the sensitivity of $T A Z$ analysis and could also detect moderate sized deletions likely missed by our approach.

Genetic heterogeneity in dilated cardiomyopathy is high with mutations in over 30 genes already linked to the phenotype. ${ }^{18,19}$ Molecular genetic testing that includes $T A Z$ sequencing for the phenotype is now supported by published guidelines and available through various laboratories. ${ }^{8,20}$ In spite of the multi-gene testing approach the assessment of both family history and phenotype remain key components of the clinical assessment process. Overall, while $T A Z$ testing is included in available molecular genetic testing panels our data indicate that the yield of TAZ testing in the absence of specific features of Barth syndrome is low and probably should not be included in first-line testing for adults with dilated cardiomyopathy. TAZ testing in select groups with clear X-linked inheritance or with concomitant neutropenia should still be considered. Furthermore, it appears that $T A Z$ mutations will not be found where the family history includes affected females. These data are useful in the genetic evaluation and counseling of families affected by adult-onset dilated cardiomyopathy and should guide initial evaluations to focus on genes more commonly implicated than TAZ. 
drome. Circulation 2001:103:1256-63.

8. Hershberger RE, Lindenfeld J, Mestroni L, et al. Genetic evaluation of cardiomyopathy - a Heart Failure Society of America practice guideline. J Card Fail 2009;15:8397.

9. Mestroni L, Maisch B, McKenna WJ, et al. Guidelines for the study of familial dilated cardiomyopathies. Collaborative Research Group of the European Human and Capital Mobility Project on Familial Dilated Cardiomyopathy. Eur Heart J 1999;20:93-102.

10. Baralle M, Baralle D, De Conti L, et al. Identification of a mutation that perturbs NF1 agene splicing using genomic DNA samples and a minigene assay. $\mathrm{J}$ Med Genet 2003;40:220-2.

11. Cortez-Dias N, Varela MG, Sargento L, et al. Left ventricular non-compaction: a new mutation predisposing to reverse remodeling? Rev Port Cardiol 2009;28:185-94.

12. D'Adamo P, Fassone L, Gedeon A, et al. The $\mathrm{X}$-linked gene G4.5 is responsible for different infantile dilated cardiomyopathies. Am J Hum Genet 1997;61:862-7.

13. Fayssoil A, Nardi O, Orlikowski D, Annane D. Cardiomyopathy in Duchenne muscular dystrophy: pathogenesis and therapeutics. Heart Fail Rev 2010;15:103-7.

14. Taylor MR, Ku L, Slavov D, et al. Danon dis- ease presenting with dilated cardiomyopathy and a complex phenotype. J Hum Genet 2007;52:830-5.

15. Plant LD, Bowers PN, Liu Q, et al. A common cardiac sodium channel variant associated with sudden infant death in African Americans, SCN5A S1103Y. J Clin Invest 2006;116:430-5.

16. Taylor MR, Slavov D, Ku L, et al. Prevalence of desmin mutations in dilated cardiomyopathy. Circulation 2007;115:1244-51.

17. Yamashita T, Hamidi Asl K, Yazaki M, Benson MD. A prospective evaluation of the transthyretin Ile122 allele frequency in an African-American population. Amyloid 2005;12:127-30.

18. Kimura A. Molecular basis of hereditary cardiomyopathy: abnormalities in calcium sensitivity, stretch response, stress response and beyond. J Hum Genet 2010; 55:81-90.

19. Taylor MR, Carniel E, Mestroni L. Cardiomyopathy, familial dilated. Orphanet J Rare Dis 2006;1:27.

20. Ghosh N, Haddad H. Recent progress in the genetics of cardiomyopathy and its role in the clinical evaluation of patients with cardiomyopathy. Curr Opin Cardiol 2011; 26:155-64. 